\title{
Comprendiendo la historia desde la comunicación: medios, medio e historia
}

\author{
Jesús Octavio Elizondo Martínez \\ (Universidad Autónoma Metropolitana Unidad Cuajimalpa, México)
}

Recibido: $30 / 4 / 2014$

Aprobado: 29/5/2014

\begin{abstract}
Resumen: Para explicar el sentido de una aproximación propiamente comunicacional a la historia, en este trabajo se emplea, principalmente, la obra del economista e historiador canadiense Harold A. Innis. Se esgrimen los argumentos que sostienen la hipótesis de que con la combinación de los enfoques histórico, mediático y cultural se logra un análisis metodológico válido para la investigación en el campo de la comunicación. Este trabajo ha sido posible gracias al apoyo del International Council for Canadian Studies, ICCS (Ottawa); la Universidad Autónoma Metropolitana Unidad Cuajimalpa (Ciudad de México), y el Consejo Nacional de Ciencia y Tecnología Conacyt/ SNI (México).

Palabras clave: ciencias de la comunicación / medios de comunicación / teoría de la comunicación / historia de los medios / Harold A. Innis / historia de Canadá
\end{abstract}

\section{Understanding history through Communication: media, social environment and history}

Summary: This paper deals with the work of Canadian economist and historian Harold A. Innis in order to analyze and elaborate on the communicational approach to history. The main hypothesis stresses that tackling the analysis of history, from historical, mediatic and cultural frameworks, provides a valid methodological approach for research in the field of communication studies. This work has been elaborated with the generous and kind support of the International Council for Canadian Studies, ICCS (Ottawa), the Metropolitan Autonomous University of Mexico (UAM-C), Cuajimalpa campus and the National Council for Science and Technology (Conacyt/SNI), Mexico.

Key words: Communication studies / mass media / theory of communication / history of media / Harold. A. Innis / history of Canada 


\section{El papel de los medios en la conformación de la historia}

It is the search for truth, not «truth» that makes men free.

H. A. INNIS

E sta investigación parte de una pregunta esencial y varias complementarias: ¿cuál es el papel que han desempeñado los medios en la conformación de la historia? Así, ¿cuál es el lugar particular de los medios en el conjunto de hechos que la historia retoma? ¿Qué hay en común entre los medios de transporte, los medios de comunicación y las tecnologías digitales a la luz de los hechos históricos?

Si por conformación entendemos identificar la colocación, la distribución de las partes que forman un conjunto (DRAE, 2012), entonces, nos interesa analizar esa distribución de elementos en el conjunto total de signos históricos con el fin de identificar el rol de los medios en el conjunto llamado historia. Aceptamos una acepción de medio como «el conjunto de circunstancias o condiciones exteriores a un ser vivo que influyen en su desarrollo y en sus actividades», así como "conjunto de circunstancias culturales, económicas y sociales en que vive una persona» (DRAE, 2012). Equivale al concepto de contexto o ecología de los medios (media ecology) en el sentido de environment - como lo usan McLuhan (1999) y Postman (1970)-. Por medios entendemos aquí, aquellas acepciones que incluyen a los medios de transporte, a los medios de comunicación preeléctricos, a los medios de comunicación de masas y a los medios de transmisión de información digital en una interpretación amplia de la definición básica de medios de comunicación: «órgano destinado a la información pública» (DRAE, 2012). Esta acepción es equivalente a mass media (media technologies, mass communication), en inglés. Emplearemos el adjetivo comunicacional en la acepción de "perteneciente o relativo a los medios de comunicación» (DRAE, 2012), al estudio de los medios de comunicación desde la teoría de la comunicación y a la historia de los medios de comunicación.

En esta investigación preferimos la definición de historia en términos formales lógicos, es decir, como el «conjunto de los sucesos o hechos políticos, sociales, económicos, culturales, etc., de un pueblo o de una nación». Queremos con esto alejarnos de la noción narratológica asociada a la historia: «narración y exposición de los acontecimientos pasados y dignos de memoria, sean públicos o privados» (DRAE, 2012). Preferimos el enfoque lógico general (conjunto de sucesos) por su implicación amplia que concuerda con una teoría del conocimiento y la significación de corte semiótico (Peirce). Nos alejamos de la idea de la historia 
como una narración por el sesgo estructuralista que implica hablar desde ese lugar, además de que hacer esto conlleva emplear metodologías propias de la lingüística (Saussure, Greimas, entre otros). A estas definiciones iremos añadiendo características específicas de diversas perspectivas teóricas coherentes entre sí.

El marco teórico referencial consiste en la dimensión propiamente mediática e histórica en la obra de Harold A. Innis (1894-1952) compilada en libros no traducidos aún al castellano: Empire and Communication, The Bias of Communication, Changing Concepts of Time. Emplearemos los conceptos «monopolio de conocimiento», sesgo temporal y sesgo espacial, equilibrio en los medios, equilibrio espacio-temporal, etc., acuñados por el canadiense. También aplicaremos aquí el método pragmaticista para dar sentido pertinente a los conceptos empleados (Charles Sanders Peirce, 1839-1914), argumentos explicados con profusión en textos publicados por el autor (Elizondo, 2012).

En el marco de la sociedad tecnificada, «técnica refiere a la totalidad de los métodos racionalmente concebidos para obtener eficiencia absoluta en cada campo de la actividad humana»
(Ellul, 1964, p. xxv). En esta investigación se empleará esta definición general, pero cabe señalar que, en ciertos casos, el énfasis podrá ser en la racionalidad, otras veces en la eficiencia o en los procedimientos, pero la definición general continuará siendo la misma. Nos alejamos así de una definición en la que técnica y tecnología son vistas únicamente como procedimientos para alcanzar un fin. La técnica no es un fenómeno aislado en la sociedad, pues está relacionado con cada aspecto de la vida del hombre moderno, influye en cada aspecto social. Dicho lo anterior, hay que preguntarse ¿cómo podemos identificar los temas clave, los problemas teóricos esenciales que presenta esta indagación, e identificar la metodología apropiada para desarrollar una investigación consistente con el objetivo planteado?

La clave para comprender el objetivo metodológico de este trabajo es que centraremos la atención en el punto de encuentro entre medios, historia y medio. Este orden de elementos responde al enfoque comunicacional empleado aquí. Este enfoque presupone que el conjunto Medios y el conjunto Medio están mediados por el conjunto Historia. 
Figura 1

Meta conjunto

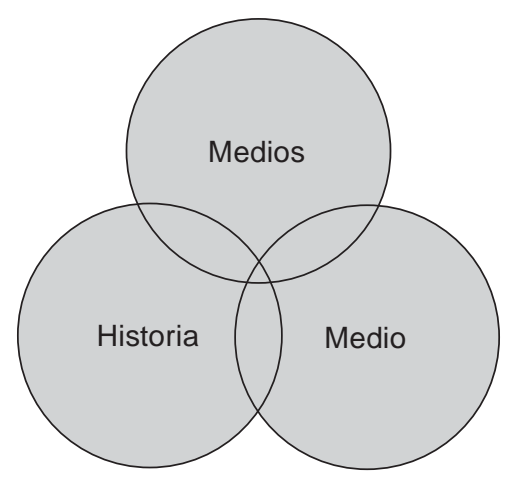

¿Cuáles relaciones podemos identificar en este esquema? Primero, estudiaremos la intersección de los Medios con el Medio (por ejemplo: el papel de los medios de comunicación en la economía, la sociedad, la cultura, y el de estos en aquellos) mediante los registros históricos (por ejemplo: archivos, bibliotecas, fototecas, etc.). De esta manera, centraremos la atención en la intersección Historia/Medios, para entonces inferir la relación Historia/ Medio (por ejemplo: los efectos de los medios en el medio). La intersección tripartita Historia/Medios/Medio será el punto nodal de la aproximación comunicacional. Segundo, haremos un estudio comunicacional de la relación entre historia y medio. No haremos aquí historia de los medios, ni historia de la economía, ni historia de la sociedad o la cultura (medio). En todo caso, emplearemos los insumos pertinentes de esas áreas de conocimiento para construir el objeto de estudio propia- mente comunicacional sin caer, a priori, en un determinismo mediático (los Medios como motores de la Historia). Tercero, hay que decir que no consideramos a los medios como motor de la historia, ni de la economía, la sociedad o la cultura (medio), sino como un factor más en la configuración de los elementos constitutivos del metaconjunto Historia + Medio + Medios. Nos interesa describir las relaciones netamente comunicacionales entre los elementos de los conjuntos para llegar, esperamos, a conclusiones pertinentes para los tres conjuntos.

Esta relación de mediación implica una semiotización de la historia para comprender su papel como signointerpretante (Peirce) o conocimiento (Kant) que brinde sentido a la relación tripartita Historia, Medios y Medio. Relación que no puede ser observada bajo la óptica bipartita de la relación sujeto-objeto (realismo ingenuo): medio/medios, medios/medio, historia/ 
medios; medios/historia, medio/historia; historia/medio. Partimos de la premisa de que cada una de estas relaciones se encuentra mediada por la Historia.

\section{Un acercamiento comunicacional a la historia}

El objetivo central de esta investigación es describir críticamente el papel de los medios en la conformación de la historia. Para lograr este objetivo abordaremos la obra del canadiense Harold A. Innis (1951) sobre el tema. Economista, político e historiador de la economía, estudió, desde una perspectiva amplia, el rol que han desempeñado los medios tanto en la historia del mundo occidental como en el caso particular de la historia de Canadá. El punto de partida de Innis es que la historia de una cultura, nación, imperio o civilización puede ser comprendida mediante el estudio y observación del desarrollo de sus redes de comercio, transporte y comunicación. Este es el punto de partida y premisa para el estudio propiamente comunicacional.

La aproximación metodológica deberá ayudar a definir un acercamiento comunicacional a la historia. Para lograr esto, las ideas fundacionales de Innis serán revisadas con el fin de ofrecer una reflexión crítica sobre el estado de la cultura y la tecnología en la actualidad: «I have attempted to suggest that Western civilization has been profoundly influenced by communication and that marked changes in communications have had important implications» (Innis, 2003, p. 3). Estas «importantes implicaciones» son el tema de esta investigación. Es decir, nos abocaremos a identificar la manera en que los cambios significativos en las comunicaciones generan implicaciones importantes en nuestra concepción de la historia.

En este trabajo, la historia es concebida como una serie de épocas separadas por la discontinuidad. Cada época se distingue por las formas dominantes de medios que absorben, registran y transforman información en sistemas de conocimiento que se encuentran en consonancia con la estructura del poder institucional de la sociedad en cuestión. De acuerdo con Innis, la interacción entre el modo de los medios y la «realidad social» crea ciertos sesgos, tendencias que influencian fuertemente los valores y la orientación cultural de la sociedad. Esas tendencias en la comunicación funcionan como un crisol a través del cual es posible identificar el carácter de una cultura, nación o civilización.

Para Innis, la estabilidad relativa de las culturas depende del balance y la proporción de sus medios. Para comenzar una investigación en este tema, sugiere que nos hagamos tres preguntas básicas: 1) ¿cómo funciona una tecnología de la comunicación específica?, 2) ¿qué insumos toma de la sociedad y de qué manera contribuye 
a ella?, y, finalmente, 3) ¿qué formas de poder incita? Posteriormente (McLuhan, 1999), estas preguntas fueron reestructuradas para dar sentido a los medios como extensiones de las facultades del hombre en cuatro preguntas o leyes de los medios. Cuando pensamos en un medio: 1) ¿qué facultad del hombre agranda o incrementa?, 2) ¿qué otra tecnología desgasta o deja obsoleta?, 3) ¿qué recupera que haya estado antes en desuso?, y 4) ¿qué invierte o cambia cuando se lo empuja hasta el límite de su potencial? Al hacer esto, podremos distinguir no solamente a la tecnología específica que nos interesa analizar (figura), sino también ubicaremos el contexto al que transforma (fondo o ground), así como la técnica que deja obsoleta y aquella práctica cultural que recupera.

Para Innis, la clave para el cambio social se encuentra en el desarrollo de los medios de comunicación. Cada medio conlleva un sesgo en los términos en que organiza y controla la información. Toda sociedad tiene necesidad de preservar y difundir su información, ya sea mediante la duración en el tiempo y/o la expansión sobre el espacio territorial. Este punto del trabajo de Innis es esencial para las ciencias de la comunicación, porque introduce la dimensión espacio-temporal en el estudio de los medios. Como hombre de su tiempo, Innis estaba al tanto de lo que sucedía en el debate académico en la entonces demócrata Escuela de Chicago de principios del siglo XX con John Dewey, Robert Park,
George Mead, Leo Löwenthal y Paul Lazarsfeld, entre otros. Entonces, los estudios sobre los medios en los Estados Unidos eran de corte empírico, orientados a la investigación de los contenidos, la información, el comportamiento del consumidor, con un énfasis en el individuo como la unidad de análisis adecuada y pertinente (Elizondo, 2010). En contraste, Innis prefirió centrar su atención en el estudio de los efectos de los medios ya no en términos de la respuesta del individuo a ciertos contenidos, sino en términos de cómo cada medio, conforme se desarrolla, reestructura patrones de interacción más amplios. Esta es la premisa básica que emplearemos en esta investigación en su sentido pragmaticista y no determinista.

\section{El cambio tecnológico y los medios de comunicación: una teoría de la historia}

Harold A. Innis hace una lectura de la historia de las civilizaciones desde la perspectiva de las industrias de la comunicación y los medios de transporte. De esta lectura surgirán dos grandes e importantes temas: el espacio y el tiempo. Por primera vez en las teorías de la comunicación, estos vectores aparecen como intrínsecos a la comprensión de la economía política de los medios de comunicación. Nos dirá que el sentido social del espacio y el tiempo se forma en los sujetos a partir -en buena medida- del tipo de medios 
de comunicación y transporte que un imperio, una sociedad o una nación desarrollen. También abordará la cuestión de la dicotomía oralidad y escritura, para abogar por el valor social de la tradición oral frente al embate de las tecnologías mecánicas, propias del pensamiento matemático y alfabetizado. Monopolio del conocimiento, medio de comunicación y el sesgo en la comunicación (también tendencia o inclinación), civilización e imperio son algunos conceptos clave que Innis empleará en su reflexión y que revisaremos en el presente trabajo.

Harold A. Innis dedicó sus esfuerzos como historiador de la economía a sustentar una teoría acerca del papel central de la comunicación y su influencia en el desarrollo de la historia humana. Esta inquietud tomó impulso durante su estancia en la Universidad de Toronto en la década de 1950 y, en especial, a partir de 1962 cuando se publicaron tres trabajos muy distintos, pero que tenían un marco teórico general que él compartía. Estos trabajos fueron La galaxia Gutenberg de Marshall McLuhan (1962), Prefacio a Platón de Eric Havelock (1963) y el ensayo titulado «Las consecuencias de la alfabeticidad» de Jack Goody e Ian Watt (1968). Si bien en aquellos años en Toronto estos académicos tuvieron muy poco contacto con Innis, sin duda, su investigación forma algo parecido a un círculo o colegio invisible que difunde las ideas de Innis acerca de la comunicación en las propias disciplinas de estos autores: en la antropología, las letras clásicas y la historia literaria (Heyer \& Crowley, 2003, p. xxi). De maneras distintas, estos trabajos se basaron en el argumento de que los modos de comunicación son fundamentales para comprender el desarrollo histórico de ciertas formas culturales y sus consecuencias en todos los ámbitos sociales. Innis elaboró una tricotomía de fases en la evolución de los medios de transporte y comunicación a lo largo de la historia: primero fue el habla y la primacía de la oralidad, como lo ha estudiado Havelock; luego la estandarización de las formas de alfabeticidad y numeración; $\mathrm{y}$, por último, la mecanización de la escritura mediante la impresión y la divulgación del libro.

\section{Harold A. Innis y el pensamiento canadiense en comunicación}

El debate filosófico europeo sobre el papel del lenguaje y el giro pragmático de la filosofía - analítica de Oxford y Viena- de la segunda mitad del siglo XX es una característica de la obra de Havelock, quien llegó de Inglaterra a Canadá, y la desarrolló en su camino a los Estados Unidos. Esta influencia fue decisiva para Harold A. Innis.

Harold Innis, renombrado economista de Toronto, podría parecer un candidato inverosímil para enzarzarse en la cuestión de oralidad y escritura; inverosímil parecía que dedicara sus energías intelectuales, durante los últimos años de una vida trágicamente 
malograda, a la exploración histórica del papel de la oralidad en las culturas humanas del pasado. Y, sin embargo, había una conexión con sus intereses profesionales, surgida de la creciente convicción de que los modos de comunicación, el «prejuicio (bias) de la comunicación» [sic], como él lo llamaba [sesgo o tendencia preferimos en este trabajo], juega un papel por lo menos igual al de la actividad económica en la formación y la dirección de la sociedad humana (Innis, 1951). (...) En las comunidades de la gente de las pequeñas ciudades entre las que se había criado veía una identidad personal y una validez del lenguaje y de la comunicación que estaban siendo erosionadas, según pensaba, por la tecnología de la prensa popular y del papel barato, de noticias instantáneas que nutrían un pensamiento instantáneo y superficial: todo ello era resultado de la alfabetización popular. Partiendo de una tensión dentro de su propio presente, la extrapoló a la historia, remontándose a los griegos, a Mesopotamia, Egipto, Asiria (Havelock, 1996, pp. 70-71).

Una característica esencial del método de investigación inniano será este enfoque comparativo en la historia, esta forma de identificar momentos de transición tecnológica e identificar patrones y relaciones similares entre estos.

Como profesional, Innis había estudiado a fondo la industria canadiense del papel y de la pasta de papel [sic] [pulpa de papel]. Como patriota, creía ver que se estaba destruyendo los bosques de su país para garantizar un efímero instante de lectura superficial en el metro de Nueva York. (...) y advertía sobre el peligro de su corrupción. En este aspecto McLuhan fue su discípulo, en cuanto estaba dispuesto a ver en la imprenta un motor del cambio social. Pero la imprenta de caracteres móviles de la que hablaba McLuhan no era la rotativa de Innis. En efecto, cuando atacaba al libro impreso y ensalzaba la liberación del libro que se había logrado gracias a la tecnología moderna y, en particular, a la tecnología electrónica, estaba colocando cabeza abajo a Innis (Havelock, 1996, p. 70).

La principal tesis inniana que dice que la cultura, la sociedad y la civilización cambian aparejadas con los cambios en los medios de comunicación. Un punto importante que tratar con detenimiento es el hecho de que McLuhan haya invertido la explicación de Innis sobre la naturaleza de la interacción entre medios y sociedad. Este aspecto es esencial para poner en la justa medida la influencia del economista sobre el crítico literario. La segunda tesis central de Innis dice que hay un sesgo en los mensajes comunicativos y este sesgo es hacia el tiempo o hacia el espacio. En su trabajo hay muestras de la sustitución del ojo por el oído en la medida que los medios de comunicación cambian, causando una transformación en la percepción. Hay que tomar en cuenta la siguiente propuesta de Innis, que resulta antitética al principal postulado de McLuhan: 
La introducción del alfabeto significó más una relación con el sonido que con la vista o más con el oído que con el ojo. Se han construido imperios con comunicaciones basadas en la vista en contraste con la organización política griega que enfatizó la discusión oral (Babe, 2000, pp. 376-377).

El trabajo de Innis es fascinante y complejo a la vez. Es fascinante, pues se propone hacer una historia de la comunicación desde el antiguo Egipto hasta la primera mitad del siglo XX. Pero su método, si bien vincula momentos clave en la historia de las civilizaciones, resulta de lo más ambicioso y a veces, al yuxtaponer momentos históricos, cae en generalizaciones abrumadoras. Además, la lectura se complica dada la oscuridad de su escritura. Por ejemplo, en el ensayo titulado Minerva's Owl (2003), Innis se da a la tarea de recrear toda la historia desde el inicio de la escritura y el cálculo por los sumerios y los egipcios, hasta la radiodifusión comercial masiva a mediados del siglo XX. Tarea monumental. Al adentrarnos en la lectura vemos que para alcanzar su objetivo, en un solo párrafo cubre la historia de China y la India. La mención del imperio romano le lleva una página o dos. La estructura es a veces dislocada y el estilo repetitivo: no hay un estilo literario agradable para el lector. Sin embargo, contiene ideas originales repartidas aquí y allá a lo largo del texto. Esta es una característica que ha sido muy comentada por los estudiosos de
Innis. Baste como muestra el siguiente párrafo:

The civilization of Greece emphasized unity of approach but Rome absorbed rhetoric and excluded science. In the East, Persian and Arabic literature excluded the influence of Greek literature but absorbed science. Pervasiveness of language becomes a powerful factor in the mobilization of force particularly as a vehicle for the diffusion of opinion among all classes. Language exposed to major incursions became more flexible, facilitated movement among classes, favored the diffusion of technology, and made for rapid adjustment (Innis, 2003, p. 11).

Muchas ideas y demasiadas generalizaciones más o menos interconectadas en un solo párrafo: las civilizaciones de Grecia y Roma, retórica y ciencia, literatura persa y árabe, el papel del lenguaje en la difusión del conocimiento entre las clases sociales, difusión y tecnología. Algunos autores (como McLuhan) han asociado el estilo particular de Innis con el de un artista que expone sus ideas - aforismos que formaban patrones reconocibles-, de tal modo que parecían conformar un gran mosaico en el que el lector debe hacer descubrimiento tras descubrimiento por sí mismo, mediante la exploración y la meditación. Asimismo, le impresionó su método. Innis no estudiaba el contenido de las estructuras - por ejemplo, los tipos de libros en viejas bibliotecas o la naturaleza de las filosofías o religiones-, sino más bien 
la existencia de bibliotecas o la predominancia de la creencia religiosa per se (Babe, 2000, p. 273). Llama la atención la estrategia mediante la cual Innis traza catálogos de detalles de donde extrae conclusiones basadas en la repentina identificación de un patrón, un modelo de aquí para más adelante. McLuhan recomienda un "patrón de reconocimiento» como estrategia para abrirse camino en una era saturada de información (Babe, 2000, pp. 273- 274).

Innis llegó al estudio de la comunicación de manera indirecta. Durante la mayor parte de su carrera profesional, se dedicó al estudio de varias industrias canadienses como el ferrocarril, el comercio de pieles, la minería y la pesca. Cuando sus investigaciones lo llevaron al tema de la producción de pulpa de celulosa y la industria del papel, tuvo la idea de dirigir sus esfuerzos hacia el estudio de «las rutas de comercio del pensamiento y la opinión pública» (Radner, 1959, p. 77). La historia económica de la imprenta, de la industria del periódico, así como todas las formas de medios de comunicación, pasaron a ser su objeto de estudio. Se abocó al análisis histórico de estos fenómenos desde los egipcios hasta mediados del siglo XX. El resultado de esto son tres libros, que juntos dejan ver una teoría de la historia que enfatiza como factor crucial el cambio tecnológico en los medios de comunicación.
Estos tres libros vieron la luz durante los primeros tres años de la década de 1950: Empire and Communications (London: Oxford University Press, 1950), The Bias of Communication (Toronto: University of Toronto Press, 1951) y Changing Concepts of Time (Toronto: University of Toronto Press, 1952). Este último fue publicado pocos meses después de su fallecimiento. Desde una perspectiva comunicacional, es posible distinguir en estos trabajos tres grandes preocupaciones que, como ejes, atraviesan y estructuran toda su obra: 1) el cambio tecnológico de los medios de comunicación como factor clave en la historia; 2) la vitalidad de la tradición oral vernácula en distintas épocas; 3) los medios masivos de comunicación y el desequilibrio que provocan en la «estabilidad» de la cultura.

\section{Historia de las comunicaciones: en busca del balance en las sociedades}

En esta sección, comentaremos cinco citas extraídas de materiales encontrados en el archivo Harold A. Innis de la Universidad de Toronto'.

Entre los papeles que dejara Innis a su muerte, estaba un manuscrito sin titular, de más de mil páginas, que, según se cree, sería retrabajado y publicado como libro. Los primeros tres capítulos fueron editados y publicados

1 Se trata de las cajas BOX B1972-0025 y BOX B72-0025/026 de University of Toronto Harold A. Innis Archives. Consultado en julio de 2012. 
bajo el título Empire and Communications (Oxford, 1950). Los materiales de los capítulos posteriores fueron empleados por Innis para ser publicados en forma de artículos y, finalmente, compendiados en The Bias of Communication (Toronto, 1951) y en Changing Concepts of Time (Toronto, 1952). Exceptuando los tres primeros capítulos, el manuscrito original que preparaba permanece intacto, tanto la parte mecanografiada como la escrita a mano. Tras la muerte de Innis, el dilema al que se enfrentaron tanto Mrs. Innis como el comité editorial de sus trabajos fue qué hacer con el manuscrito. Si bien no estaba listo para su publicación, no consideraron correcto dejarlo archivado, así es que se decidió que, para que pudiera ser consultado por los especialistas, sería microfilmado. Esta tarea fue encargada a Mrs. Jane Ward ${ }^{2}$.

Para el economista, el estudio de las industrias de la comunicación debería ser fundamental. No es así, y sin embargo Innis lo apunta desde antes del nacimiento de la disciplina que estudia el fenómeno de la comunicación. Como se verá en la cita siguiente, esta idea es comprensible en la medida que se trata de su objetivo empírico, existencial, si se me permite la expresión, así como una estrategia esencial en su agenda política dentro de la universidad:

To the economist the study of communication industries is of fundamental importance. The character of competition varies with the commerciality of knowledge. The sensitivity of economic life and the possibilities of disturbances to equilibrium are dependent to an important extent on the press ${ }^{3}$.

Desde el punto de vista de un economista, resulta pertinente considerar los elementos constitutivos del proceso de comunicación como insumos o recursos naturales para la producción de bienes y servicios. De aquí que la idea de un equilibrio, un balance entre los elementos del proceso de producción, distribución y consumo sea un objetivo deseable. Innis conocía el trabajo que los investigadores prominentes en los Estados Unidos realizaban en el campo de la comunicación, tal y como consta en las reseñas que hizo de obras de la autoría de Leo Löwenthal y Paul Lazarsfeld: «Mr. Leo Löwenthal pleads for an interest of sociologist in the 'imagination in print' (...). Mr. Paul Lazarsfeld is concerned with ways and means of reducing skittishness of the mass communications industries

2 A History of Communications. An Incomplete and Unrevised Manuscript by H. A. Innis. Microfilmed for private circulation. Editorial note (original en inglés). Material original disponible en la caja BOX 017(01)-(08) de University of Toronto Harold A. Innis Archives. Consultado en julio de 2012.

3 Disponible en las cajas B72-0025/023 Fólder (29) P. 9 de University of Toronto Harold A. Innis Archives. Consultado en julio de 2012. 
at the hands of research workers» ${ }^{4}$. Si consideramos lo anterior y las relacionamos con otros hechos, como su estrecha vinculación con la Universidad de Chicago, podemos decir que Innis proponía un enfoque distinto, novedoso y distante para el estudio del fenómeno de la comunicación: partir de la historia de la economía (historia económica) hacia la lógica de la economía de los medios. Esta lógica de los medios es, según él, el equilibrio utópico, ideal y deseable entre la dimensión espacial (geográfica y territorial) y la temporal. Esta hipótesis la pondrá a prueba a lo largo de sus estudios sobre la industrias del papel y la prensa escrita, característicos de su primera etapa.

En una conferencia impartida en la Universidad de New Brunswick en mayo de 1944, Innis reúne en un párrafo varias ideas que, de manera sintética, dejan ver su concepción de la importancia que el balance juega en la manera en que los monopolios de conocimiento operan en momentos históricos. Es un párrafo característico de su escritura abigarrada y de su método comparativo para el análisis histórico:
Her [the University of Paris] traditions and her interest demand an obsession with balance and perspective-an obsession with the Greek tradition of the humanities. The search for truth assumes a constant avoidance of extremes and extravagance. Virtue is in the middle way. There are no cures. Always we are compelled to be sceptical of the proposal to cure the world ills. We cannot tolerate the dominance of any individual or of any group. The University of Paris checked in France the virulence which characterized the Inquisition in Spain. In our time it must resist the tendencies to bureaucracy and dictatorship of the modern State, the intensification of nationalism, the fanaticism of religion, the evils of monopoly in commerce and industry ${ }^{5}$.

Esto es importante, pues nos permite identificar las raíces del concepto de balance en la tradición griega (y no únicamente como lo hemos dicho anteriormente). Si bien en este párrafo se refiere al papel de la universidad como un monopolio del conocimiento que se ha enfrentado y debe enfrentarse a monopolios del conocimiento eclesiástico entonces, y comercial actualmente, es de suponer que la comparación es válida para otras situaciones histó-

4 Disponible en las cajas 72-0025/023 Fólder (29) de University of Toronto Harold A. Innis Archives. Consultado en julio de 2012.

5 Address to graduates: A Plea for the University Tradition. Address to convocation of the University of New Brunswick, May, 1944. In The Dalhousie Review, 298-305. Proofs for edition. P. 299-300. Disponible en las cajas B1972-0025 y BOX B72-0025/026 de University of Toronto Harold A. Innis Archives. Consultado en julio de 2012. 
ricas similares. Además de esta, resaltan varias ideas más. Por ejemplo, es sobre el papel de la Inquisición española que se formó una divergencia radical entre Innis y McLuhan. Bien conocido es este pasaje, pues si bien hay afinidades identificables en los métodos comparativos de ambos autores, no hubo entre ellos la empatía ideológica que podría llevar a construir una relación más allá de los límites de la academia. Destaca el enfoque de un economista librecambista que prefiere el modelo económico centrado en los productos y el consumo, que uno (como el de la URSS) centrado en el trabajador y el Estado. Pero es el papel que la universidad debe tener ( $\mathrm{y}$ ha tenido, como es el caso) en el juego del balance lo que llama la atención como cierre retórico del párrafo: «En nuestro tiempo [la universidad] debe resistirse a seguir la tendencia hacia la burocracia y la tiranía del Estado moderno, la intensificación del nacionalismo, el fanatismo de la religión y los demonios del monopolio en el comercio y la industria». Estas ideas son tan vigentes entonces como ahora. Si el equilibrio aparece ya como esencial en la tradición griega, luego será un elemento característico de la lógica del historiador de tipo «agustiniano». Con motivo del obituario de Charles Norris Cochrane (1889-1945), escribirá en 1945:
History written from the philosophical background of classicism differs sharply from history written from the Augustinian point of view with its emphasis on will, personality, and unpredictability. [...] A society dominated by Augustinian will produce a fundamental different type of historian, who approaches his problem from the standpoint of challenge and profess, from classicism with its emphasis on cyclical change and of classicism assumed the tendency of equilibrium 6 .

Llama la atención la manera en que Innis emplea el concepto de balance y equilibrio, como característica, en este caso, de la universidad, pero como baluarte de la tarea histórica que media entre puntos de vista radicales, que propone soluciones totales, sea el marxismo o el keynesianismo. Esta tarea resulta esencial para la sobrevivencia de la civilización. Así, en las universidades recae esta tarea; en el monopolio de conocimiento que es la universidad.

The university must deny the finality of any of the conclusions of the social sciences. It must steadfastly resist the tendency to acclaim any single solution of the world's problems at the risk of failing to play its role as balancing factor in the growth of civilization. The Marxist solution, the Keynesian solution, or any solution, cannot be accepted as final if the universities are to continue and civilization is to survive.

6 Disponible en las cajas B1972-0025 y BOX B72-0025/026 de University of Toronto Harold A. Innis Archives. Consultado en julio de 2012. 
(...) It is possible that an application of demand and supply curves may assist in determining their limitations, but the character of civilizations suggest that the problem is philosophical and perhaps beyond their power to solve ${ }^{7}$.

\section{Los monopolios del conocimiento: imperio, nación y comunicaciones}

El estudio profundo de la economía política inspira el interés por la historia de las comunicaciones o historia de los medios. Este interés dará cuerpo al campo que más tarde será llamado ciencias de la comunicación (Communication Studies). Si lo que nos interesa es reconocer el papel que los medios tienen y han tenido como mediadores de las relaciones económicas, entonces, una aproximación interdisciplinaria puede ser pertinente, pues el impacto que esta relación tiene en el medio es inmenso y complejo. Al hacer esto habremos abierto la posibilidad de un amplio proyecto histórico, donde estarán integradas la economía política y la comunicación. En el estilo provocador característico de Innis, para describir lo que ahora llamamos sociología del conocimiento, emplea una paradoja: «We need a sociology or a philosophy of the social siciences and particular economics, an economic history of economic history» (Heyer, 2003, p. 38).

\section{Los sesgos espaciales y temporales en las comunicaciones: un aporte esencial a la teoría de la comunicación}

En el artículo «The Bias of Communication», Innis establece dos tendencias en la formación del conocimiento. Una sociedad oral, tribal y con pocos textos escritos sobre papiro o papel será una sociedad que crea medios de comunicación y soportes de la comunicación que tienen como fin preservar los mensajes a lo largo del tiempo (time-binding). Este orden, por lo general eclesiástico, es característico de sociedades descentralizadas, donde el diálogo y la vida comunal, así como la memoria, son clave para la preservación del conocimiento. Las sociedades industrializadas, que bajo la lógica de la mecanización y la producción en serie han logrado expandirse sobre vastos territorios, han creado artefactos tecnológicos que llevan los mensajes desde un centro hasta la periferia y viceversa, como en el caso de los imperios. Este sesgo hacia la expansión territorial (space-building) es caracte-

7 Innis, H. A. (1944). Political Economy in the Moder State. Reprinted from Proceedings of the American Philosophical Society, 87 (4), p. 341. Disponible en las cajas B1972-0025 y BOX B720025/026 de University of Toronto Harold A. Innis Archives. Consultado en julio de 2012. 
rístico de los medios de comunicación electrónicos. Además de estos dos ejes que hemos mencionado, Innis acuña el concepto de monopolio del conocimiento para definir una característica del poder. A lo largo del tiempo, en diferentes momentos históricos, el poder ha creado monopolios del conocimiento para asegurarse un lugar en el desarrollo de nuevos saberes, la predicción del futuro y el control político. Al observar los soportes de los mensajes de la comunicación en una sociedad, podemos ver el tipo de tendencia que hay en ella, en sus creencias, en sus propósitos y en su manera de entender el mundo. Al abordar el tema de los medios de transporte y comunicación, Innis tenía en mente a la civilización occidental. Para él los alcances tecnológicos de una civilización serían el centro de atención para deducir el tipo de sociedad que los creara. Las tecnologías y la manera de hacer cosas con ellas surgen de las civilizaciones y, por lo tanto, manifiestan las preocupaciones prototípicas (Babe, 2000, p. 61) $\mathrm{y}$ los patrones de pensamiento de una sociedad particular. Además, las tecnologías tienen siempre algún impacto en las civilizaciones que las crean y en las que entran en contacto con ellas; por eso, son un elemento importante para comprender la evolución de las sociedades. Las tecnologías son el vehículo por el cual las civilizaciones se expanden y entran en contacto entre sí. Por lo anterior, las tecnologías, en general, pueden ser comprendidas co- mo medios de comunicación, incluso en el caso de aquellas que no han sido creadas con el fin comunicativo. Esta característica semiótica de los artefactos tecnológicos hace muy rico el estudio de los efectos de la tecnología en los sujetos que entran en contacto con ella. Innis aborda a profundidad este asunto en su texto A History of the Canadian Pacific Railway (1923). Ahí define al ferrocarril como la causa que explica la fuerza y el carácter de la civilización europea. Gracias a este invento fue posible, entre otras cosas, el desarrollo de la industrialización mediante el flujo masivo de bienes y materias primas. Asimismo, fue un efecto en la medida que se puede tomar como el resultado del conocimiento que las sociedades industrializadas habían alcanzado para entonces. Además, para Innis es importante señalar el papel del medio que ayudó a difundir y diseminar en el territorio norteamericano las ideas de la civilización europea. Lo anterior fue posible de dos maneras. Primero, creó mensajeros que llevaban consigo nuevos mensajes (pasajeros e inmigrantes con nuevas ideas y maneras de trabajar y de vivir), los cuales entraron en contacto con los habitantes originarios. Segundo, el ferrocarril fue un mensaje en sí mismo; un mensaje de serialización, de mercantilización y de uso de la energía característico de la época en Europa. Fue un signo puesto en contacto con los nativos, que transmitía estos valores y que eventualmente transformaría sus vidas y el territorio (Elizondo, 2009). 
¿Por qué es importante el concepto de tiempo en el estudio de los medios de comunicación? Hay que considerar al tiempo como nuestra más importante innovación: una innovación del pensamiento abstracto. La concepción del tiempo corre paralelamente al uso de herramientas y de signos abstractos. Umberto Eco, en su texto introductorio a la semiótica (Eco, 1989), explica, mediante un interesante ejemplo, que es posible atribuir funciones a los objetos en un momento prelingüístico y que las palabras fueron creadas para fijar en la comunidad, mediante la comunicación, la función que puede ser atribuida o reconocida en diversos objetos. Eco refiere a un australopiteco que utiliza una piedra para descalabrar el cráneo de un mono; si bien aún no hay cultura en ese hecho, cabe notar que un elemento de la naturaleza ha sido transformado en utensilio. Habrá cultura cuando un ser pensante establezca una nueva función de la pie$\mathrm{dra}$, la denomine como una piedra que sirve para algo y la reconozca como la piedra que corresponde a la función asignada y que tiene un nombre. Eco nos dice que utilizar una piedra por primera vez no es cultura, sin embargo, establecer qué y cómo la función puede repetirse en el tiempo y transmitir esta información, sí lo es. En el momento que se produce la comunicación entre los hombres, uno supone que el signo lingüístico está presente, pero no siempre es así. El emisor puede comunicar la función del objeto, incluso sin denominarlo verbalmente, sino tan solo mostrándolo. Sin duda, la denominación de tal función como «arma» o «rompe cráneos» asegura que el conocimiento de esa experiencia se transmita de sujeto a sujeto mediante la comunicación en el tiempo, y este hecho sí que puede ser considerado como parte de la cultura. Así, en el momento en que el posible uso de la piedra ha sido conceptualizado, la propia piedra se convierte en signo concreto de su uso virtual (Eco, 1989, p. 29).

Uno de los argumentos centrales de los estudios que toman como punto de partida los medios de comunicación y su impacto cultural es que una cultura particular es, en gran medida, el reflejo del tipo de tendencia temporal que adopta. Las culturas, como los individuos que las componen, están ligadas a su propia concepción del tiempo, y esto define en gran parte su especificidad y personalidad. Los estudios del tema han propuesto seis dimensiones temporales, que interactúan constantemente entre sí en el contexto social: cada evento, pensamiento o situación se puede definir en términos de su estructura secuencial, duración, planeación, tasa de repetición, sincronización y perspectiva temporal (Rifkin, 1989, p. 59). La manera en que una sociedad escoge cómo emplear cada una de estas dimensiones temporales determina la orientación global de esa cultura. Así, con el fin de interactuar exitosamente en sociedad, debemos saber qué es lo que se espera de 
nosotros en cada momento y en cada circunstancia. Gran parte del proceso de aprendizaje, como hemos visto anteriormente, está destinado a enseñarnos el orden apropiado en el que las cosas deben hacerse. Asimismo, aprendemos cuánto tiempo deben durar las acciones que realizamos, desde una visita social de cortesía, pasando por el tiempo culturalmente razonable para un duelo por el fallecimiento de un ser querido, hasta la entrega de un reporte en el trabajo. Las culturas varían mucho en el tiempo que asignan a diversas actividades, y estas diferencias son el objeto de estudio de antropólogos y sociólogos. La planeación general de una actividad puede darse a partir del referente del entorno natural, considerando las estaciones y planeando en función de ellas. De manera específica, en el caso de la construcción de una casa, se deberá recurrir a los calendarios laborales oficiales, a los límites de entrega de materiales para construcción y a los precios vigentes, todo intentando gastar lo menos posible y ahorrar el máximo de energía. Es pertinente observar las diferencias culturales en la concepción de la velocidad y su relación con el tiempo. Según reporta Rifkin (1989, p. 73), en las sociedades agrarias, el tiempo es concebido como un fenómeno cíclico, repetitivo y sagrado. En el caso de los estudiantes norteamericanos entrevistados en la década de 1980, su concepción del tiempo estaba fuertemente determinada por la noción de velocidad y rapidez. Esta concepción del tiempo es fundamental para la interacción social, pues la sincronización entre sus miembros debe ser compartida en todos los niveles de acción colectiva de una comunidad, ciudad, cultura o nación. Finalmente, la perspectiva temporal nos deja ver el sesgo de una cultura en particular y permite observar diferencias y similitudes con otras culturas. En esta perspectiva temporal, la cultura norteamericana ha estado siempre más orientada al presente y al futuro (Rifkin, 1989, p. 77) que, por ejemplo, la mexicana, que constantemente hace referencias al pasado para comprender el presente.

Innis influyó como ningún otro académico canadiense de su generación en muchos y diversos campos de pensamiento e investigación: historia, economía, sociología, ciencia política y derecho ${ }^{8}$. A esta lista habrá que añadir las disciplinas que surgieron posteriormente y que, en cierta manera, comparten el método inniano de investigación: los estudios culturales, la ciencia de la comunicación, la teoría y metodología para la investigación de la comunicación, la historia de los medios, la historia de la tecnología y,

8 Sidney E. Smith, President of the University of Toronto, in Convocation Hall, Nov., 10, 1952, con ocasión del homenaje póstumo a Innis. Disponible en B72-00003/005 Fólder (41) de University of Toronto Harold A. Innis Archives. Consultado en julio de 2012. 
por supuesto, los estudios que tienen como objeto de estudio la ecología de los medios (media ecology).

\section{Conclusiones}

En la construcción de su gran proyecto History of Communications, Harold. A. Innis trabajó sobre la base de tres ejes fundamentales. Primero, la ecuación oralidad-escritura. El papel de la escritura $-\mathrm{y}$, por lo tanto, el del alfabeto- como una primera tecnología que permitió el surgimiento de un pensamiento crítico, analítico-filosófico, dejando a la oralidad en la tradición de la creencia en el mito. Segundo, el método diacrónico comparativo en la historia. Identifica momentos de transición tecnológica que marcan una crisis en la comunicación humana desde la Antigüedad hasta la Edad Contemporánea, para establecer una relación entre ellos. Tercero, la naturaleza de las tecnologías de la comunicación y su relación con el sentido social del tiempo y el espacio. Esta característica es fundamental para comprender el tipo de pensamiento y sociedad a la que pertenecen ciertas tecnologías. Estudiando esto, es posible observar los sesgos espacio-temporales, en la medida que el ser humano crea hábitos de acción y de apropiación.

El estudio comparativo de momentos de transición tecnológica permite a Innis presentar un panorama de las repercusiones que se dieron en la ma- nera de ver el mundo, a partir de las crisis que se produjeron en periodos históricos clave, para entender el desarrollo de la civilización occidental. Estas crisis de transición, que interesaron a Innis, implicaron una transformación en la forma de ver el mundo (Weltanschauung) en los hombres y mujeres que entraron en contacto con las nuevas tecnologías de su tiempo. La crisis de comunicación de cada transición, al ser estudiada en comparación con las otras, permitió establecer una correlación entre ellas, más allá de la relación de similitud. Pero su método, al vincular momentos clave en la historia de las civilizaciones, resulta de lo más ambicioso y, a veces, al exponer muy sucintamente la forma en que diferentes momentos históricos comparten patrones en su estructura, cae en generalizaciones abrumadoras. El reconocimiento de estos patrones es, sin duda, el elemento metodológico más rescatable de su obra. Con base en esto, es que se sostiene una aproximación propiamente comunicacional a la comprensión de la historia. Esto en virtud de que el campo de estudio de la comunicación ha sido consolidado en los últimos cincuenta años, mediante la definición de sus objetos de estudio. Cabe señalar aquí que la tendencia del campo de comunicación ha sido simplificar la cuestión mediante el enfoque histórico-cronológico de la historia de los medios de comunicación o la historia de las tecnologías de la información y la comunicación. 
Es deseable que un enfoque como el planteado en este trabajo incida en el campo disciplinario.

\section{Referencias}

Babe, R. (2000). Canadian Communication Thought. Ten Foundational Writers. Toronto: UTP.

Babe, R. (2011). Media, Structures and Power. The Robert E. Babe Collection. Edición de Edward Como. Toronto: UTP.

Dewey, J. (1915). Democracy and Education. Nueva York: Macmillan.

Eco, U. (1989). La estructura ausente. Barcelona: Lumen.

Elizondo, J. O. (2009). La escuela de comunicación de Toronto. Comprendiendo los efectos del cambio tecnológico. México D. F.: Siglo XXI Editores.

Elizondo, J. O. (2010). El pensamiento pragmatista norteamericano en comunicación. Revista Iberoamericana de Comunicación, 19, 29-38.

Elizondo, J. O. (2012). Signo en acción. El origen común de la semiótica y el pragmatismo. México D. F.: Paidós Mexicana, Col. Comunicación.

Ellul, J. (1964). Le vouloir et le faire: recherches éthiques pour les chrétiens. Ginebra: Labor et Fides.

Havelock, E. (1996). La musa aprende a escribir. Reflexiones sobre oralidad y escritura desde la Antigüedad hasta el presente. Barcelona: Paidós.
Heyer, P. (2003). Harold Innis. Lanham, MD: Rowman \& Littlefield Publishers.

Heyer, P., \& Crowley, D. (2003). Introduction. En Harold A. Innis, The Bias of Communication, pp. X-XXVI. Toronto: UTP.

Innis, H. A. (1923). A History of the Canadian Pacific Railway. Londres: P. S. King and Son.

Innis, H. A. (1950). Empire and communications. Londres: Oxford University Press.

Innis, H. A. (1952). Changing concepts of time. Toronto: UTP.

Innis, H. A. (2003) [1951]. The Bias of Communication. Toronto: UTP.

Innis, H. A. (2003). Minerva's Owl. En H. A. Innis. The Bias of Communication. Toronto: UTP.

McLuhan, M., \& McLuhan, E. (1999). Laws of media: The new science. Toronto: UTP.

Postman, N. (1970). The reformed English curriculum. In High school 1980: The shape of the future in American secondary education. Edited by Alvin C. Eurich, 160-168. New York: Pitman.

Radner, S. (1959). The communication concepts of Harold Innis. College Composition and ommunication, NCTE, 10 (2), pp. 77-80.

Rifkin, J. (1989). Time Wars. The Primary Conflict in Human History. Nueva York: Touchstone. 